\title{
THE STRUCTURE OF Z-RELATED SETS
}

\author{
FRANCK JEDRZEJEWSKI, TOM JOHNSON
}

\begin{abstract}
The paper presents some new results on Z-related sets obtained by computational methods. We give a complete enumeration of all Z-related sets in $\mathbb{Z}_{N}$ for small $N$. Furthermore, we establish that there is a reasonable permutation group action representing the Z-relation.
\end{abstract}

Keywords: Interval vector, Patterson function, Z-relation, Homometry, Combinatorics, Music Theory.

The concept of Z-relation has been discussed in a systematic way by Allen Forte [6. In the 1940s, the concept appears in crystallography under the name of "homometric point sets" in the paper of A.L. Patterson [14. In these years, homometric sets have been extensively studied by mathematicians and crystallographers. R.K. Bullough pointed out some general theorems 2 until J. Rosenblatt rephrased and extended the problem in a new algebraic framework. In music theory, the problem was studied by S. Soderberg in 1995 [17, and by J. Goyette in 2012 [7]. J. Mandereau et al. 11], 12] gave a new starting point in 2011.

\section{Z-RELATION AND HOMOMETRY}

A pitch class set is a set of pitches where octaves are equivalent, and enharmonically equivalent pitches are identified. In music theory, the Z-relation links two different set classes with the same interval vector. $N$-tone equal temperament is represented by pitch classes $\mathbb{Z}_{N}=\{0,1, \ldots, N-1\}$. For a given set $A \subset \mathbb{Z}_{N}$, the interval vector measures the number of ways the interval $n$ can be spanned between members of $A$.

$$
\operatorname{iv}(A)(n)=\operatorname{ifunc}(A, A)(n)=\#\{(a, b) \in A \times A, b-a=n\}
$$

The interval function of two sets $A$ and $B$, introduced by David Lewin in [10, is the number of times any $k$ in $A$ has its $n$-transpose in $B$, namely:

$$
\operatorname{ifunc}(A, B)(n)=\sum_{k} \mathbf{1}_{A}(k) \mathbf{1}_{B}(n+k)
$$

where $\mathbf{1}_{A}$ is the indicator function defined by $\mathbf{1}_{A}(k)=1$ if $k \in A$ and $\mathbf{1}_{A}(k)=0$, otherwise. If the sets $A$ and $B$ are equal, the interval function is the interval vector. The interval content is the set of first digits of the interval vector, $(\operatorname{iv}(A)(1),, . ., \operatorname{iv}(A)([N / 2]))$, except that the last one is divided by 2 if $N$ is even. For example, the interval vector of the set $\{0,1,3,4,7,9\}$ is $(6,2,2,4,3,2,4,2$, $3,4,2,2)$, and its interval content is $(2,2,4,3,2,2)$.

Two sets $A$ and $B$ of $\mathbb{Z}_{N}$ are said to be $Z$-related if they have the same interval content:

$$
\left(A \mathcal{Z}_{N} B\right) \Leftrightarrow \operatorname{ic}(A)=\operatorname{ic}(B)
$$

In other words, $A$ and $B$ share the same interval function, or the same interval vector. Since transposing or inverting do not change the interval content, we get a lot of trivially $Z$-related sets. To avoid trivial cases, we consider set classes up to transposition and inversion, that is under the action of the dihedral group. The first well-known example was given by Lino Patterson in 1944: 


\section{$\{0,3,4,5\} \mathcal{Z}_{8}\{0,4,5,7\}$}

If we draw the two sets of points on a circle representing $\mathbb{Z}_{8}$, we will see that the distances between the points are the same but the points are in different arrangements. The two sets share the same interval content $i c=2121$ (meaning from left to right that there are 2 pairs with distance 1, 1 pair with distance 2, 2 pairs with distance 3 , and 1 pair with distance 4 ). In dealing with finite abelian groups $\mathbb{Z}_{N}$, it will be convenient to use polynomial notation. The polynomial representing the set $A$ of $\mathbb{Z}_{N}$ is the polynomial whose exponents are the elements of $A$ :

$$
A(x)=\sum_{a \in A} x^{a}
$$

The reflection of $A$ is the polynomial whose exponents are the inversion $I(A)$ of $A$ $\bmod N$

$$
A^{*}(x)=\sum_{b \in I(A)} x^{b}
$$

$I$ denotes the inversion $I(x)=-x \bmod N$. By definition, the Patterson function of the set $A$ is the autocorrelation function given by the convolution product

$$
F(x)=A * A^{*}(x)=A(x) A\left(x^{-1}\right)
$$

modulo $\left(x^{N}-1\right)$. The Patterson function is an equivalent of the interval function, since we have the following result.

Proposition 1. The coefficients of the Patterson function are equal to the components of the interval function.

$$
A * A^{*}(x)=\sum_{c_{i} \in \operatorname{ifunc}(A)} c_{i} x^{i}
$$

Two sets $A$ and $B$ of $\mathbb{Z}_{N}$ are said to be homometric if they have the same Patterson function. The musical concept of Z-related sets coincides with the crystallographic notion of homometric sets.

Example. For $N=12$ and $A=\{0,2,3,5\}$, it is easy to verify the previous result.

$$
\begin{aligned}
A(x) & =1+x^{2}+x^{3}+x^{5}, \quad A^{*}(x)=1+x^{10}+x^{9}+x^{7} \\
F(x) & =A(x) A\left(x^{-1}\right)=4+x+2 x^{2}+2 x^{3}+x^{5}+x^{7}+2 x^{9}+2 x^{10}+x^{11} \\
\text { ifunc }(A) & =(4,1,2,2,0,1,0,1,0,2,2,1)
\end{aligned}
$$

\section{SOME GENERAL THEOREMS}

In $\mathbb{Z}_{N}$, the set $A$ is trivially homometric under the action of the dihedral group, that is under all inversions $I_{n}(A)$ and transpositions $T_{n}(A)$, where inversions are defined by $I_{n}(x)=-x+n \bmod N$, and transpositions are defined by $T_{n}(x)=$ $x+n \bmod N$.

Moreover, let $A$ and $B$ be subsets of $\mathbb{Z}_{N}$, then $A$ and $B$ are said to be trivially homometric if they belong to the same orbit under the action of the dihedral. If $A$ and $B$ are homometric but not trivially homometric, then they are strictly homometric. Thus the Z-relation is equivalent to strict homometry.

In 1944, Patterson [14] established the following two results:

(1) If two subsets of a regular $N$-gon are homometric then their complements are.

$$
A \mathcal{Z}_{N} B \Leftrightarrow A^{c} \mathcal{Z}_{N} B^{c}
$$

(2) Every $N$-point subset of a regular $2 N$-gon is homometric to its complement. 
In terms of music theory, the $N$-note set $A$ of the $2 N$-tone equal temperament is in Z-relation with its complement $A^{c}$. For example, for $N=4$, the 4 -note set $A=\{0,1,3,5\}$ of $\mathbb{Z}_{8}$ and its complement $A^{c}=\{2,4,6,7\}$ are in Z-relation since

$$
\operatorname{ic}(A)=(1,2,2,1)=\operatorname{ic}\left(A^{c}\right)
$$

If we consider now the multiplication by an integer $m$ modulo $N$, it is easy to show that the Z-relation is stable by multiplication. More precisely, let $A$ be a set in $\mathbb{Z}_{N}, m$ an integer such that $\operatorname{gcd}(m, N)=1$ and $m \neq 1, N-1$. Then the interval content ic $(A)$ is in general different from $\operatorname{ic}\left(M_{m}(A)\right)$, where $M_{m}$ denotes the multiplication by $m$ modulo $N, M_{m}(x)=m x \bmod N$. We have

$$
A \mathcal{Z}_{N} B \Longrightarrow M_{m} A \mathcal{Z}_{N} M_{m} B
$$

Example. In the usual temperament $(N=12)$, since the two complementary sets $A=\{0,1,2,3,5,6\}$ and $B=A^{c}=\{0,1,2,3,4,7\}$ are in Z-relation with interval content ic $(A)=433222$, we get a new homometric pairs by mutliplying each set by 5. Thus, $M_{5} A=\{0,1,3,5,6,10\}$ and $M_{5} B=\{0,3,5,8,10,11\}$ are Z-related with a new interval content equal to $\operatorname{ic}\left(M_{5} A\right)=233242$.

Remark. Unfortunately, not all Z-related pairs are coming from complement nor multiplication, $A \mathcal{Z}_{N} A^{c}$ or $A \mathcal{Z}_{N} M_{m} A$. For example, for $N=18$, the sets $A=\{0,1,2,3,4,7,8,14,16\}$ and $B=\{0,1,2,3,5,6,7,9,13\}$ are Z-related but $B$ is neither the complementary set of $A$, nor a multiple set of $A\left(B \neq M_{k} A\right.$ for $k=5,7,11,13)$.

By adding the transposed set to the initial set, we get some new theorems:

Theorem 2. Let $A$ and $B$ be two Z-related sets in $\mathbb{Z}_{N}$, and $T_{N}$ the transposition in $\mathbb{Z}_{2 N}\left(T_{N}(x)=x+N \bmod 2 N\right)$, then we have

$$
A \mathcal{Z}_{N} B \Longrightarrow\left(A \cup T_{N} A\right) \mathcal{Z}_{2 N}\left(B \cup T_{N} B\right)
$$

Or more generally,

Theorem 3. Let $A$ and $B$ be two Z-related sets in $\mathbb{Z}_{N}$, and $T_{j}$ the transpositions in $\mathbb{Z}_{N m}$, then we have

$$
A \mathcal{Z}_{N} B \Longrightarrow\left(A \cup T_{N} A \cup \ldots \cup T_{N(m-1)} A\right) \mathcal{Z}_{N m}\left(B \cup T_{N} B \cup \ldots \cup T_{N(m-1)} B\right)
$$

The same can be done with the multiplication.

Theorem 4. Let $A \mathcal{Z}_{N} B$ and $m$ be an integer such that $\operatorname{gcd}(N, m)=1$ and $m \neq 1, N-1, M_{m}$ be the multiplication and $T_{j}$ the transpositions in $\mathbb{Z}_{N m}$, then we have

$$
\left(M_{m} A \cup T_{1} M_{m} A \cup \ldots \cup T_{m-1} M_{m} A\right) \mathcal{Z}_{N m}\left(M_{m} B \cup T_{1} M_{m} B \cup \ldots \cup T_{m-1} M_{m} B\right)
$$

Example. Starting from the homometric pair $\{0,1,3,4\} \mathcal{Z}_{8}\{0,1,2,5\}$, the multiple relation of $M_{3} A=\{0,3,9,12\}$ and $M_{3} B=\{0,3,6,15\}$ leads to the new pair:

$$
\{0,1,2,3,4,5,9,10,11,12,13,14\} \mathcal{Z}_{24}\{0,1,2,3,4,5,6,7,8,15,16,17\}
$$

In 2008, O'Rourke, Taslakian and Toussaint [13] gave a new procedure for generating homometric pairs called the Pumping Lemma, based on adding points around isospectral vertices (see their paper for details). We propose another new procedure starting with not one, but two homometric pairs. In the general case, this procedure is lengthy and will be published elsewhere. We consider here an example. Choose two suitable pairs. For example $\{0,1,3,4,6\} \mathcal{Z}_{10}\{0,1,2,4,7\}$ and $\{0,1,4,5,7\} \mathcal{Z}_{12}\{0,1,2,5,8\}$. Place the first of each pair on a circle of 10 points 
(Fig. 1, left), and add the second of each pair on the circle as shown on Fig. 1 (center). On each of the two points connected by a diameter, we can add $k$ points, leading to the new relation (Fig. 1 right)

$$
\{0,1,3+k, 4+k, 6+k\} \mathcal{Z}_{10+2 k}\{0,1,2,4+k, 7+k\}
$$

Remark that the number of added points is always even, even if they are several diameters.
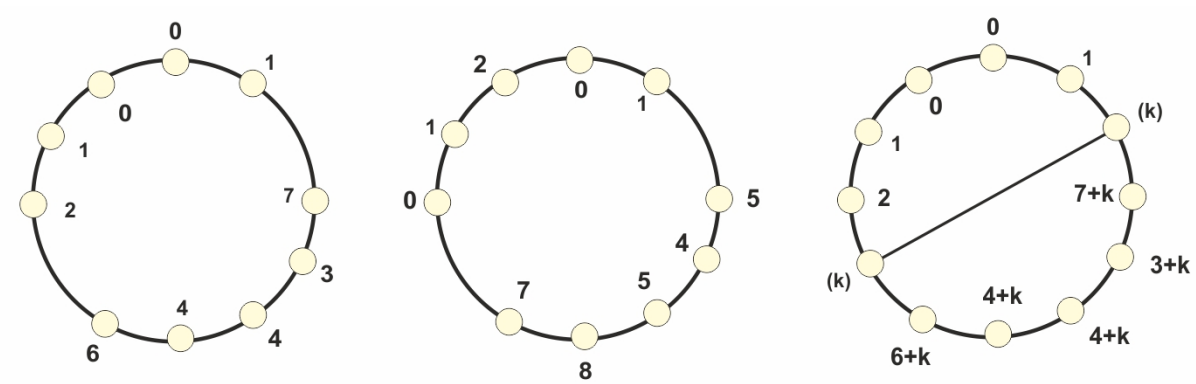

Figure 1. The two interlaced pairs

The aim of the pumping lemma or of this procedure is to find a way of establishing general relations, such as this one, established empirically. For $n \geq 5$, we have

$$
\begin{aligned}
& \{0,1, n-2, n-1, n+1\} \mathcal{Z}_{2 n}\{0,1,2, n-1, n+2\} \\
& \{0,1,2, n-2, n+1\} \mathcal{Z}_{2 n}\{0,1,3, n-1, n\}
\end{aligned}
$$

Rosenblatt gave a complete classification of Z-related pairs of length 4 :

If $A \mathcal{Z} B$ with $\operatorname{card}(A)=\operatorname{card}(B)=4$ then $A$ and $B$ are of the following two types:

(i) In $\mathbb{Z}_{4 n}, \exists a \in\{1,2, \ldots, n-1\}, n \geq 2$,

$$
A=\{0, a, a+n, 2 n\} \mathcal{Z}_{4 n} B=\{0, a, n, 2 n+a\}
$$

(ii) In $\mathbb{Z}_{13 n}$,

$$
A=\{0, n, 4 n, 6 n\} \mathcal{Z}_{13 n} B=\{0,2 n, 3 n, 7 n\}
$$

But the question of finding the classification of all Z-related pairs for $\operatorname{card}(A) \geq 5$ is still open (see [1, 4]).

\section{ENUMERATION OF Z-RELATED SETS}

Enumeration of Z-related sets was studied by Patterson, and later by others, including Bürger [3] and Chieh [5]. Patterson [14] was the first to demonstrate the existence of $t$-uples of homometric sets. For $N \leq 12$, homometric sets are well known. Some progress has been made for "complementary" homometric pairs with $N$ even. But from $N \geq 16$, non-complementary homometric pairs appear. In [9] David Lewin rediscovered, and brought to the attention of the music-theoretic community, the existence of the Z-triples for $N=16$, and Jon Wild [18] has previously entabulated all homometric tuplets for $N \geq 31$, with tuplets of 15 -note sets with as many as 16 members in $\mathbb{Z}_{30}$. But enumeration is still an open problem. Our computation led to the following table. For each $k$-note sets, the table gives the number of (distinct) interval vectors in $\mathbb{Z}_{N}$ for which non-trivial homometric tuples exist (sets are considered up to inversion and transpositions). For example, for $N=16$, 
there are 31 non-trivial homometric tuples of $k=6$ notes. (In fact, 28 homometric pairs +3 homometric triplets).

\begin{tabular}{c|cccccccccc}
\hline$k \backslash N$ & 8 & 10 & 12 & 13 & 14 & 15 & 16 & 17 & 18 & 19 \\
\hline 4 & 1 & 0 & 1 & 1 & 0 & 0 & 2 & 0 & 0 & 0 \\
5 & - & 3 & 3 & 0 & 6 & 5 & 10 & 0 & 14 & 0 \\
6 & - & - & 15 & 2 & 6 & 25 & 31 & 16 & 62 & 21 \\
7 & - & - & - & - & 48 & 10 & 44 & 24 & 134 & 57 \\
8 & - & - & - & - & - & - & 180 & 52 & 150 & 90 \\
9 & - & - & - & - & - & - & - & - & 572 & 156 \\
\hline
\end{tabular}

Italics indicate the existence of tuples $(t>2)$. For $N=12$, there are 19 homometric pairs $(1+3+15)$.

\begin{tabular}{c|ccccc}
\hline$k \backslash N$ & 20 & 21 & 22 & 23 & 24 \\
\hline 4 & 2 & 0 & 0 & 0 & 3 \\
5 & 22 & 0 & 20 & 0 & 31 \\
6 & 98 & 96 & 60 & 33 & 275 \\
7 & 191 & 220 & 335 & 110 & 676 \\
8 & 535 & 282 & 575 & 429 & 2532 \\
9 & 565 & 1062 & 1425 & 814 & 5112 \\
10 & 2106 & 613 & 1550 & 1144 & 7715 \\
11 & - & - & 7390 & 1375 & $?$ \\
12 & - & - & - & - & $?$ \\
\hline
\end{tabular}

The first triple appears for $N=16$ and length 6 :

$$
\{0,1,2,4,6,9\}, \quad\{0,1,2,4,9,14\}, \quad\{0,1,3,5,7,8\}
$$

and the first quadruple is obtained for $N=18$ and length 9 (in fact, there are 54 quadruples). There are three quintuples for $N=24, k=10$, one sextuple for $N=24, k=7$ and one octuple for $N=24, k=9$ : a very impressive property of the quarter-tone universe.

$$
\begin{array}{lll}
\{0,1,2,4,6,9,12,16,17\} & \{0,1,2,4,6,9,14,17,18\} & \{0,1,2,4,8,9,12,14,17\} \\
\{0,1,2,4,9,10,14,17,22\} & \{0,1,2,4,9,14,16,17,20\} & \{0,1,2,6,9,10,12,14,17\} \\
\{0,1,3,5,7,8,13,16,17\} & \{0,1,3,5,8,9,13,15,16\} &
\end{array}
$$

\section{Group Action for Z-RELAtion}

Since our interest is to give a fast algorithm of finding all homometric sets, one way is to understand how they are organized and to answer the question: Is there a non-trivial group action representing the Z-relation (such that the orbits are the equivalence classes of the Z-relation)? If you look at a subgroup of the linear group, you certainly do not find a solution, as shown by Mandereau et al. 12. But if you look at a subgroup of the permutation group $\mathfrak{S}_{N}$, you will find a nice non-trivial solution. Considering the set $\mathcal{B}$ of all transpositions and inversions for a given $N$ of all homometric sets of length $k$, the automorphism group of $\mathcal{B}$ is clearly a solution of the problem. In other words, the group is a subgroup of $\mathfrak{S}_{N}$ whose action on the power set of $\mathbb{Z}_{N}$ stabilizes $\mathcal{B}$. To compute this automorphism group, the idea is to use a well-known object in combinatorial block design known as the Levi graph $\Gamma$. Denoting the homometric sets $\mathcal{B}=\left\{B_{1}, \ldots, B_{u}\right\}$, the vertex set of the Levi graph is 
$V=\left\{0,1,2, \ldots, N-1, B_{1}, \ldots, B_{u}\right\}$. The $N$ first elements of $V$ have one color and the $\mathcal{B}$ elements have a second color. The edge set is defined by:

$$
\left\{\left(i, B_{j}\right): i \in B_{j}, i=0,1, \ldots, N-1\right\}
$$

As is well known, the automorphism group of the Levi graph is the same as the automorphism group of $\mathcal{B}$. Thus, the problem is to compute the automorphism group of a vertex-colored graph, which is done by a $\mathrm{C}$ program. The information is provided in the form of a set of generators, the size of the group and the orbits of the group. After this computation, the difficulty is to give a nice representation of the orbits of the group. Let's have a look at some examples.

Example 1. The simplest example is to compute the automorphism group of the homometric sets for $N=8$. As we know, there is only one Z-related pair, but 16 transpositions and inversions. The pair

$$
\{0,1,2,5\} \mathcal{Z}_{8}\{0,1,3,4\}
$$

leads to a set $\mathcal{B}$ of 16 elements. The automorphism group is generated by four generators $a=(1,3)(2,6)(5,7), b=(1,5)(3,7), c=(0,1)(2,7)(3,6)(4,5), d=(2,6)(3,7)$. The group has only one orbit, and is represented on Fig 2 . The inner circle (dihedral class of the homometric set $\{0,1,2,5\})$ is linked by permutation $d$ to the outer circle (dihedral class of the homometric set $\{0,1,3,4\}$ ).

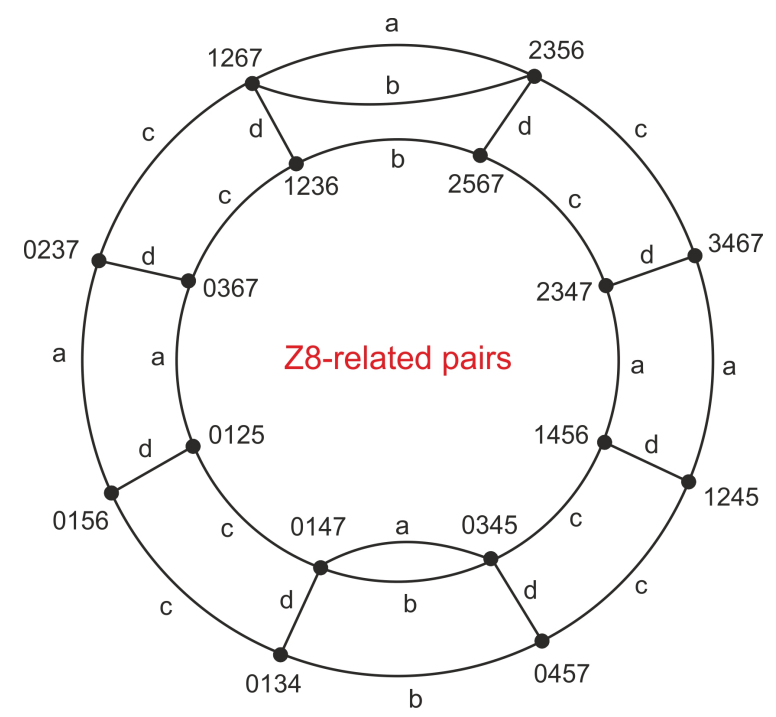

FIGURE 2. Group of $\mathrm{Z}_{8}$-related pairs

Example 2. For $N=12$, the automorphism group of the 48 homometric sets generated by the pair $\{0,1,3,7\},\{0,1,4,6\}$ of length 4 , has six generators.

$$
\begin{aligned}
a & =(3,9), b=(4,10), c=(5,11) \\
d & =(2,5)(8,11), e=(1,2)(4,5)(7,8)(10,11) \\
f & =(0,1)(3,4)(6,7)(9,10)
\end{aligned}
$$


Its representation is a rather complicated graph. The automorphism group of the 108 homometric sets of length 5 has three generators:

$$
\begin{aligned}
& a=(1,5)(2,10)(4,8)(7,11) \\
& b=(1,7)(3,9)(5,11) \\
& c=(0,1)(2,11)(3,10)(4,9)(5,8)(6,7)
\end{aligned}
$$

Tom Johnson 8 demonstrated that the homometric sets of length 5 can be graphed with less than six transformations, as one can see in the concentric circles of Fig. 3 .

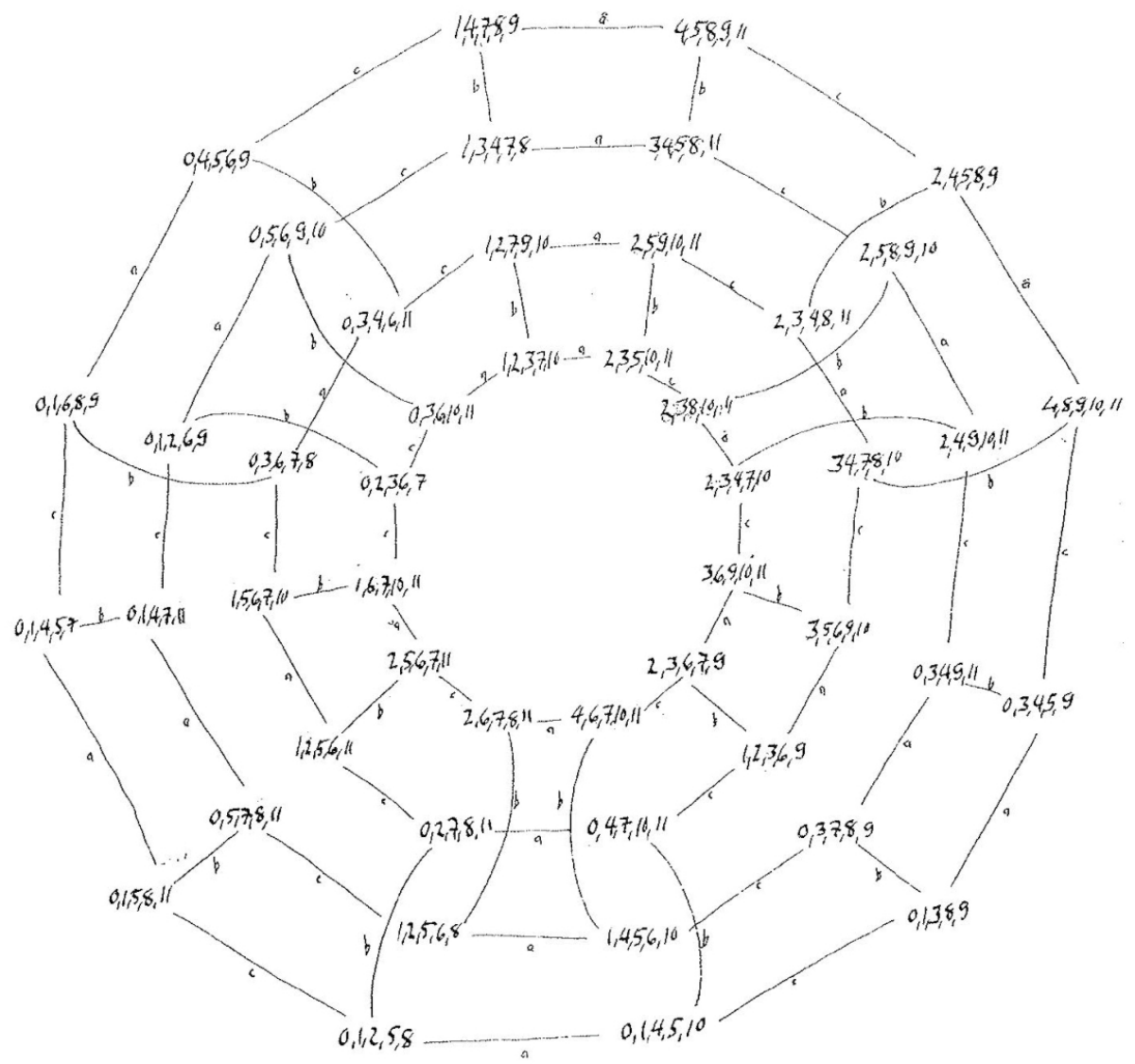

Figure 3. Tom Johnson's drawing (Orbit of the Group of $\mathrm{Z}_{12^{-}}$ related pairs of length 5)

The structure of this graph appears 6 times in the 19 orbits of the automorphism group of the 552 homometric pairs of length 6 whose generators are

$$
\begin{aligned}
& a=(2,10)(3,11)(4,8)(5,9) \\
& b=(1,3)(2,10)(4,8)(5,11)(7,9) \\
& c=(0,1)(2,3)(4,5)(6,7)(8,9)(10,11)
\end{aligned}
$$


Tom Johnson has experimented with new transformations in a more musical way. Starting with the 48 homometric four-note chords, he computed the twodimensional network of Table 1, described thus: For horizontal transformations the note contained both in the M2 (Major second) and in the m3 (minor third) moves a tritone. For odd vertical transformations the two notes of the m3 move one place away from the $\mathrm{m} 2$. For even vertical transformations, the notes of the M3 approach one another, becoming a M2, or the two notes of the M2 separate into a M3.

$\begin{array}{llll}15 \mathrm{ab} & 57 \mathrm{ab} & 457 \mathrm{~b} & 145 \mathrm{~b} \\ 059 \mathrm{~b} & 569 \mathrm{~b} & 356 \mathrm{~b} & 035 \mathrm{~b} \\ 068 \mathrm{~b} & 0568 & 0256 & 026 \mathrm{~b} \\ 067 \mathrm{a} & 0467 & 0146 & 016 \mathrm{a} \\ 1679 & 1367 & 0137 & 0179 \\ 1578 & 1257 & 127 \mathrm{~b} & 178 \mathrm{~b} \\ 2478 & 1248 & 128 \mathrm{a} & 278 \mathrm{a} \\ 2368 & 0238 & 0289 & 2689 \\ 2359 & 239 \mathrm{~b} & 389 \mathrm{~b} & 3589 \\ 1349 & 139 \mathrm{a} & 379 \mathrm{a} & 3479 \\ 034 \mathrm{a} & 049 \mathrm{a} & 469 \mathrm{a} & 346 \mathrm{a} \\ 24 \mathrm{ab} & 48 \mathrm{ab} & 458 \mathrm{a} & 245 \mathrm{a}\end{array}$

Table 1 - The complete group of 48 homometric four-note chords

By considering transformations in musical terms, Tom Johnson opens new ways of understanding homometric relationships and defines an automorphism group at the same time.

\section{Conclusion}

The aim of this paper was to consider homometric sets and to compute the number of interval vectors for each value of $N$. We establish some new theorems helping us to compute Z-related sets. Moreover, we show that there is a reasonable group representing all Z-related sets of a given length, for a given $\mathrm{N}$ and compute this group explicitly for $N$ less than 12 . However, the question of the enumeration of homometric sets remains open.

\section{ACKNOWLEDGEMENT}

Submitted to MCM 2013. The final publication will be publish by Springer Lectures Notes in Computer Sciences and available at link.springer.com. http: //link.springer.com/bookseries/558.

\section{REFERENCES}

[1] Althuis T.A., GöBel F. Z-related Pairs in Microtonal Systems. Memorandum No. 1524, University of Twente, The Netherlands, 2000, accessed from http://doc.utwente.nl/65712/1/1524.pdf on March. 26, 2013.

[2] Bullough R.K. "On Homometric Sets. I. Some General Theorems", Acta Crystallographica 14: 257-268.

[3] Buerger M. J.: "Exploration of cyclotomic point sets for tautoeikonic complementary pairs". Zeitschrift für Kristallographie 145 (1977) 371-411.

[4] Callender C. Hall R. "Crystallography and the structure of z-related sets". Paper given at the annual meeting of the Society for Music Theory in Nashville, TN, 2008. Handout accessed from myweb.fsu.edu/ccallender/z-relationhandout.pdf on March. 26, 2013.

[5] CHien C. "Analysis of cyclotomic sets", Zeitschrift für Kristallographie 150 (1979) 261-277.

[6] Forte A. The Structure of Atonal Music. Yale University Press, second edition 1977.

[7] Goyette J.S. The Z-Relation in Theory and Practice, Ph.D. thesis, University of Rochester, New York, 2012. 
[8] Johnson T., Jedrzejewski F. Looking at Numbers, Birkhauser, 2013 (to appear).

[9] Lewin D. On extended Z-triples. Theory and Practice 7 (1981) 38-39.

[10] Lewin D. Generalized Musical Intervals and Transformations. Yale University Press, 1987.

[11] Mandereau J. et al. "Z-Relation and Homometry in Musical Distributions", Journal of Mathematics and Music 5 (2) (2011) 83-98.

[12] Mandereau J. et al. "Discrete Phase Retrieval in Musical Structures", Journal of Mathematics and Music 5 (2) (2011) 99-116.

[13] O'Rourke J., Taslakian, P., Toussaint G., "A pumping lemma for homometric rhythms", Proceedings of the 20th Canadian Conference on Computational Geometry (2008) 121-123.

[14] Patterson A.L. "Ambiguities in the X-Ray Analysis of Crystal Structures", Physical Review, 65 (5-6) (1944) 195-201.

[15] Quinn I. "General Equal-Tempered Harmony (Parts II and III)." Perspectives of New Music 45 (1) (2007) 4-63.

[16] Rosenblatt J. "Phase Retrieval", Communications in Mathematical Physics 95 (1984) 317343.

[17] Soderberg S. "Z-Related Sets as Dual Inversions", Journal of Music Theory, 39 (1) (1995) $77-100$.

[18] WILD J. Enumerating set-classes and Z-related tuplets in equal temperaments of up to thirtyone notes per octave. Unpublished graduate seminar paper, McGill University (1996) 